\section{AVALIAÇÃO DA EXPERIÊNCIA DE CÁRIE E QUALIDADE DE VIDA RELACIONADA À SAÚDE BUCAL DE ESCOLARES}

\author{
Evaluation of dental caries experience and oral health-related \\ quality of life in schoolchildren
}

\author{
Evaluación de la experiencia de caries y calidad de vida \\ relacionada con la salud bucal de escolares
}

\begin{abstract}
RESUMO
Objetivo: Avaliar a relação entre experiência de cárie, qualidade de vida relacionada à saúde bucal (QVRSB) e fatores socioeconômicos em escolares de rede municipal. Métodos: Este estudo, de corte transversal, realizado em um município paulista a partir de um levantamento de saúde bucal do ano de 2012, incluiu 142 escolares com 12 anos completos para avaliação da QVRSB por meio do Child Perceptions Questionnaire $\left(\mathrm{CPQ}_{11-14}\right)$ e de fatores socioeconômicos (escolaridade dos pais, renda, número de cômodos e número de pessoas que habitam o domicílio). A experiência de cárie foi avaliada e expressa pelo índice CPOD e ceo-d (número de dentes cariados, perdidos e obturados na dentição permanente e decídua, respectivamente). A análise consistiu de estatística descritiva, uso dos testes Qui-quadrado, Mann-Whitney e correlação de Spearman. Resultados: Do total, 58,5\% (n=83) dos escolares apresentaram experiência de cárie $(\mathrm{CPOD}+$ ceo- $\mathrm{d} \geq 1)$, os quais também apresentaram maiores escores na percepção global em saúde bucal $(2,6 \pm 0,9$ x $2,1 \pm 0,8)$, na escala total $(33,0 \pm 22,6$ x $21,9 \pm 14,5)$ e nos domínios bem-estar emocional $(11,4 \pm 8,6$ x 6,6 45,8$)$ e bem-estar social $(7,7 \pm 8,2 \times 4,4 \pm 4,9)$ quando comparados àqueles sem experiência de cárie. Observouse também correlação positiva significativa entre o número de pessoas que habitavam o domicílio e o índice CPOD/ceo-d ( $\mathrm{r}=0,2670 ; \mathrm{p}=0,003)$. Conclusão: A experiência de cárie relacionou-se com uma percepção negativa da saúde bucal, principalmente nos aspectos emocional e social, e com o número de pessoas que habitavam o domicílio.
\end{abstract}

Descritores: Criança; Cárie Dentária; Qualidade de Vida.

\section{ABSTRACT}

Objective: To assess the relationship between caries experience, oral health-related quality of life (OHRQLL) and socioeconomic factors in children from public municipal schools. Methods: This cross-sectional study was part of an oral health survey conducted during the year of 2012 and included 142 children aged 12 years to assess the OHRQoL using the Child Perceptions Questionnaire $\left(C P Q_{11-14}\right)$ and the socioeconomic factors (parental education, family income, number of rooms and number of household members). Caries experience was assessed and expressed by DMFT and dmft indexes (number of decayed, missing and filled permanent and deciduous teeth, respectively). Statistical analysis consisted of descriptive statistics, Chi-squared test, Mann-Whitney's test and Spearman's correlation test. Results: In all, $58.5 \%(n=83)$ of the children had caries experience $(D M F T+d m f t \geq 1)$ and also presented higher scores in the overall perception of oral health $(2.6 \pm 0.9 \times 2.1 \pm 0.8)$, in the total scale $(33.0 \pm 22.6 \times 21.9 \pm 14.5)$ and in the emotional well-being (11.4 $\pm 8.6 \times 6.6 \pm 5.8)$ and social well-being (7.7 $\pm 8.2 \times 4.4 \pm 4.9)$ domains when compared to those without caries experience. It was also noted a significant positive correlation between the number of people living in the household and the DMFT/dmft indexes ( $r=0.2670, p=0.003)$. Conclusion: Caries experience was related to a negative perception of oral health-particularly in the emotional and social aspects - and to the number of people living in the household.

\section{Artigo Original}

1) Coordenação de Saúde Bucal Carapicuíba (SP) - Brasil

2) Faculdade de Odontologia de Piracicaba UNICAMP - Piracicaba (SP) - Brasil

3) Universidade Federal de São Paulo UNIFESP - Diadema (SP) - Brasil 


\section{RESUMEN}

Objetivo: Evaluar la relación entre la experiencia de caries, la calidad de vida relacionada con la salud bucal (CVRSB) y los factores socioeconómicos de escolares de la red municipal. Métodos: Este estudio de corte transversal realizado en un municipio se São Paulo a partir de datos secundarios sobre salud bucal del año de 2012, incluyó 142 escolares de 12 años completos para la evaluación de la CVRSB a través del Child Perceptions Questionnaire $\left(C P Q_{11-14}\right)$ y de los factores socioeconómicos (escolaridad de los padres, renta, número de cómodos y número de personas que viven en el domicilio). La experiencia de caries fue evaluada y expresa por el CPOD y ceo-d (número de dientes cariados, los perdidos y los obturados en la dentición permanente y decidua, respectivamente). El análisis se dio por la estadística descriptiva, el uso de las pruebas de Cui-Cuadrado, MannWhitney y la correlación de Spearman. Resultados: Del total, el $58,5 \%(n=83)$ de los escolares presentaron la experiencia de caries $(C P O D+c e o-d \geq 1)$ los cuales también presentaron mayores

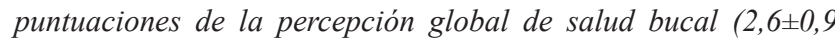
$x 2,1 \pm 0,8)$, en la escala total $(33,0 \pm 22,6 \times 21,9 \pm 14,5)$ y en los dominios bienestar emocional (11,4 $18,6 \times 6,6 \pm 5,8)$ y el bienestar social $(7,7 \pm 8,2 \times 4,4 \pm 4,9)$ al compararlos a los que no tuvieron la experiencia de caries. Se observo también la correlación positiva y significativa entre el número de personas que vivian en el domicilio $y$ el indice CPOD/ceo-d ( $r=0,2670 ; p=0,003)$. Conclusión: $L a$ experiencia de caries se relacionó con una percepción negativa de la salud bucal, en especial para los aspectos emocional y social y con el número de personas que vivían en el domicilio.

Descriptores: Niño; Caries Dental; Calidad de Vida.

\section{INTRODUÇÃO}

A cárie dentária ainda é um problema de saúde pública em todo o mundo, sendo sua ocorrência associada a fatores socioeconômicos, em função da dificuldade ou impossibilidade de acesso aos serviços públicos ${ }^{(1)}$, o que resulta em desvantagem para a população menos favorecida $^{(2)}$. De acordo com o último levantamento epidemiológico em saúde bucal realizado no Brasil ${ }^{(3)}$, na região Sudeste, a experiência de cárie dentária diminuiu ao longo dos anos, mas foi encontrada em 51,5\% das crianças com 12 anos, o que demonstra que há ainda demanda de investimentos nesse setor. Grande parte da população brasileira tem dificuldade de acesso aos serviços odontológicos, e os problemas bucais, como a cárie dentária, podem ter um impacto negativo no bem-estar das crianças e seus familiares ${ }^{(4,5)}$.

Ao longo dos anos, a saúde bucal vinha sendo avaliada por meio de critérios exclusivamente clínicos, os quais não permitiam a determinação do real impacto dos problemas bucais no dia a dia dos indivíduos. A necessidade de se determinar a repercussão integral de alterações presentes na cavidade bucal levou ao desenvolvimento dos instrumentos de avaliação da qualidade de vida relacionado à saúde bucal (QVRSB), que têm sido utilizados com maior frequência em pesquisas da área odontológica ${ }^{(6)}$.

Dessa forma, a avaliação da qualidade de vida e da percepção individual no seu contexto cultural, seus valores no meio em que vive, sua relação com os seus objetivos, expectativas, parâmetros e conceitos, vem sendo incluída na avaliação clínica, como forma de priorizar tratamentos e implementar estratégias de saúde bucal ${ }^{(4,7,8)}$. O interesse pela qualidade de vida e saúde bucal em crianças vem aumentando, já que as desordens orais provavelmente apresentam efeito negativo na qualidade de vida das $\operatorname{mesmas}^{(9)}$.

A versão brasileira do Child Perception Questionnarie (CPQ) é um instrumento de medida de QVRSB que mensura o impacto da saúde bucal das crianças nos sintomas orais, limitações funcionais, bem-estar emocional e social ${ }^{(4,10)}$, e quanto maior o escore, maior o impacto percebido ${ }^{(10)}$.

A saúde influencia positiva ou negativamente a qualidade de vida de um indivíduo, e o agravo à saúde bucal pode proporcionar restrições nas suas atividades cotidianas ou no seu bem-estar, como dor, desconforto e distúrbios de fonação e mastigação ${ }^{(11)}$. Estudos prévios conduzidos no Brasil encontraram associação entre experiência de cárie, renda familiar, escolaridade da mãe e impactos na QVRSB de crianças tanto de escolas públicas ${ }^{(1,12,13)}$ quanto privadas $^{(1,14)}$.

Embora a prevalência de cárie dentária tenha apresentado declínio nos últimos anos, a literatura mostra que existem grupos populacionais mais propensos à doença ${ }^{(15)}$. A relação de interdependência entre hábitos saudáveis, saúde e qualidade de vida mostra que as ações no âmbito da educação em saúde, sejam elas educativas, preventivas ou curativas, são instrumentos de transformação social, propiciando a reformulação de hábitos e atitudes ${ }^{(16)}$.

Dessa forma, vê-se a necessidade de aprofundar o conhecimento a respeito da relação que pode existir entre a experiência de cárie, a percepção de qualidade de vida relacionada à saúde da criança e a condição socioeconômica em que vive que possa subsidiar práticas de promoção e o planejamento na atenção à saúde. Sendo assim, o objetivo deste estudo foi avaliar a relação entre experiência de cárie, qualidade de vida relacionada à saúde bucal (QVRSB) e fatores socioeconômicos em escolares de rede municipal.

\section{MÉTODOS}

Trata-se de um estudo de corte transversal realizado a partir de um levantamento de saúde bucal no município 
de Carapicuíba-SP, ocorrido durante o ano de 2012, que envolveu escolares da rede pública municipal com 12 anos de idade completos.

O município de Carapicuíba está inserido na Região Metropolitana de São Paulo e possui 387.622 habitantes; tem taxa de analfabetismo de 4,36\%, e o Produto Interno Bruto (PIB) per capita é de R\$10.602,78, colocando-o na $606^{\mathrm{a}}$ posição dos 645 municípios do estado de São Paulo ${ }^{(17,18)}$.

Para o levantamento de saúde bucal realizado pelo município, selecionaram-se escolares a partir de um universo de 6.383 crianças matriculadas com 12 anos completos em 38 escolas de nível fundamental. Sete escolas foram sorteadas, de diversas regiões do município, e a partir da listagem de alunos, selecionaram-se os nomes das crianças de quatro em quatro para a amostra (amostra sistemática), as quais foram avaliadas por duas cirurgiãsdentistas do município, devidamente treinadas, totalizando 320 escolares avaliados. A idade de 12 anos é adotada internacionalmente como parâmetro básico para uso do indicador ${ }^{(19)}$.

Após a anuência da Coordenadoria de Saúde Bucal de Carapicuíba e da aprovação do projeto pelo Comitê de Ética em Pesquisa da Universidade Federal de São Paulo, passouse à seleção da amostra para o presente estudo. Os pais ou responsáveis foram contatados e convidados a participar, sendo enviados, pelas crianças, os Termos de Consentimento Livre e Esclarecido e de Assentimento, uma ficha de anamnese para levantamento de dados demográficos e um questionário de qualidade de vida relacionado à saúde bucal (QVRSB), todos impressos. Houve retorno positivo de 183 escolares e incluíram-se apenas aqueles que retornaram os formulários assinados e preenchidos na sua totalidade, excluindo-se os que retornaram fichas e/ou questionários incompletos. Sendo assim, o presente estudo obteve amostra final de 142 escolares com 12 anos completos (crianças não referenciadas para tratamento odontológico).

Coletaram-se os dados demográficos da população estudada por meio da ficha de anamnese adotada pelo Departamento de Odontologia Infantil da Faculdade de Odontologia de Piracicaba (UNICAMP), que foi entregue aos pais/responsáveis pelas próprias crianças, no retorno aos seus domicílios. Os pais/responsáveis devolveram as fichas preenchidas à escola, mas lembretes foram necessários, a fim de obter o maior número possível no prazo de um mês após o envio. Investigaram-se: dados pessoais, históricos pré-natal, natal e pós-natal da criança, e fatores socioeconômicos familiares. Dentre os fatores socioeconômicos, verificou-se: a) escolaridade dos pais/ responsáveis (ensino fundamental, ensino médio, ensino superior/completo ou incompleto); b) renda familiar mensal em reais; c) número de cômodos do domicílio; d) número de pessoas que habitavam o domicílio.

O levantamento de saúde bucal foi realizado por duas cirurgiãs-dentistas do município de Carapicuíba, devidamente treinadas e calibradas, no próprio ambiente escolar, em uma sala de aula reservada e utilizandose espelho bucal, sonda de ponta romba, além de equipamentos de proteção individual (gorro, máscara, jaleco e luvas descartáveis). Na avaliação da experiência de cárie, utilizaram-se os índices CPOD e ceo-d (total de dentes cariados, perdidos/esfoliados e obturados) para dentes permanentes e decíduos, respectivamente ${ }^{(19)}$. Dessa forma, formaram-se dois grupos: G1) com experiência de cárie: ao menos um dente cariado, perdido ou obturado $(\mathrm{CPOD}+$ ceo- $\mathrm{d} \geq 1)$; G2) sem experiência de cárie: dentes hígidos e ausência de perda precoce $(\mathrm{CPOD}+\mathrm{ceo}-\mathrm{d}=0)$.

Para avaliar a QVRSB, utilizou-se o instrumento Child Perceptions Questionnaire $\left(\mathrm{CPQ}_{11-14}\right)$, traduzido e validado para a população brasileira ${ }^{(9)}$. Esse instrumento avalia o impacto da condição bucal da criança em quatro domínios da saúde - sintomas bucais, limitações funcionais, bemestar emocional e bem-estar social -, em 37 itens. As duas primeiras questões do $\mathrm{CPQ}_{11-14}$ são referentes à percepção global da saúde bucal e bem-estar geral, com opções de resposta em escala Likert de 4 pontos. As demais questões também são medidas em escala Likert de 4 pontos $(0=$ nunca; $1=$ uma ou duas vezes; $2=$ algumas vezes; $3=$ =várias vezes; 4=todos os dias ou quase todos os dias). A pontuação total é obtida pela soma dos escores de todas as questões. Quanto maior a pontuação, maior o impacto na qualidade de vida da criança. $\mathrm{O}$ instrumento $\mathrm{CPQ}_{11-14}$ foi respondido pela própria criança (autorrelato), em sala reservada, sob supervisão da pesquisadora, após explicações previamente definidas a todos os participantes.

$\mathrm{Na}$ análise estatística dos dados coletados, utilizouse o pacote estatístico BioEstat 5.3 (Mamirauá, Belém, PA, Brasil), considerando-se um p-valor $<0,05$. O teste de normalidade Kolmogorov-Smirnov foi utilizado para se verificar a distribuição das variáveis. Para aquelas que apresentaram desvio da distribuição normal, utilizaram-se testes não paramétricos (Mann-Whitney e teste de correlação de Spearman). A estatística descritiva consistiu de médias, desvios-padrão, medianas e porcentagens. A proporção de sexo entre os grupos G1 e G2 (com e sem experiência de cárie) foi verificada por meio do teste Qui-quadradopartição. Utilizando-se o teste Mann-Whitney, realizouse a comparação dos escores obtidos em cada domínio e na escala total entre os grupos. Uma matriz de correlação foi obtida entre as variáveis socioeconômicas e o índice CPOD+ceo-d (pelo teste de correlação de Spearman).

As informações obtidas na presente pesquisa associadas à identidade do participante não foram reveladas 
nem consultadas por terceiros, sendo apenas utilizadas para fins estatísticos e científicos, resguardando-se a total privacidade e anonimato. Por se tratar de estudo conduzido em parceria com a Secretaria de Saúde de Carapicuíba, as crianças com diagnóstico de cárie foram referenciadas para tratamento na rede municipal de saúde. O projeto da presente pesquisa obteve aprovação do Comitê de Ética em Pesquisa da Universidade Federal de São Paulo (UNIFESP; 16984113.9.0000.5505).

\section{RESULTADOS}

Dos 142 escolares avaliados, 58,5\% $\quad(n=83)$ apresentaram experiência de cárie. A Tabela I apresenta as frequências encontradas para sexo e experiência de cárie na amostra avaliada. A proporção de sexo masculino e feminino não diferiu significantemente entre os grupos $(\mathrm{p}=0,9082)$.

A Tabela II apresenta os dados descritivos obtidos na avaliação da QVRSB, em que se podem observar os escores de cada domínio, escore total, bem como os itens de percepção global de ambos os grupos. Foi encontrada diferença significativa $(\mathrm{p}=0,0029)$ no escore total do $\mathrm{CPQ}_{11}$. ${ }_{14}$ quando comparados os grupos com e sem experiência de cárie.

A avaliação de cada domínio separadamente mostrou que os escores dos domínios bem-estar emocional e social apresentaram diferença significativa entre os grupos $(p=0,0012$ e $p=0,0051$, respectivamente). $O$ grupo com experiência de cárie apresentou maiores escores de percepção global em saúde bucal em relação às crianças sem experiência de cárie ( $\mathrm{p}=0,0013)$. No aspecto bem-estar geral, não se observou diferença significativa $(\mathrm{p}=0,0540)$.

A Tabela III mostra os coeficientes de correlação obtidos entre as variáveis socioeconômicas e o número de dentes cariados, perdidos/esfoliados e obturados. Dentre os coeficientes obtidos e de interesse ao estudo, observouse correlação positiva significativa entre o número de pessoas que habitavam o domicílio e o índice CPOD/ceo-d $(\mathrm{r}=0,2670 ; \mathrm{p}=0,0030)$.

Tabela I - Frequência [n (\%)] do gênero e média ( \pm DP) de CPOD/ceo-d da amostra avaliada. Carapicuíba-SP, 2012.

\begin{tabular}{lccc}
\hline \multicolumn{4}{c}{ Grupos } \\
\hline & Com experiência de cárie & Sem experiência de cárie & Total \\
& $\mathbf{8 3}(\mathbf{5 8 , 5})$ & $\mathbf{5 9}(\mathbf{4 1 , 5 )}$ & $\mathbf{1 4 2}(\mathbf{1 0 0})$ \\
\hline Sexo* & $40(48,2)$ & $27(45,8)$ & $67(47,2)$ \\
Masculino & $43(51,8)$ & $32(54,2)$ & $75(52,8)$ \\
Feminino & $3,7(2,4)$ & - & $2,1(2,6)$ \\
CPOD/ceo-d & & -
\end{tabular}

DP: desvio padrão; CPOD/ceo-d: número de dentes cariados, perdidos/esfoliados e obturados

* $\mathrm{p}=0,9082$ (teste Qui-quadrado)

Tabela II - Média ( \pm DP) dos escores dos domínios, escore total e itens de percepção global do Child Perception Questionnaire $\left(\mathrm{CPQ}_{11-14)}\right.$ de acordo com os grupos. Carapicuíba-SP, 2012.

\begin{tabular}{lccc}
\hline $\mathbf{C P Q}_{11-14}$ & Número de itens & $\begin{array}{c}\text { Com experiência de cárie } \\
(\mathbf{n = 8 3})\end{array}$ & $\begin{array}{c}\text { Sem experiência de cárie } \\
(\mathbf{n}=\mathbf{5 9})\end{array}$ \\
\hline Total [0-148] & 37 & $33,0(22,6)^{*}$ & $21,9(14,5)^{*}$ \\
Domínios & & & \\
Sintomas orais [0-24] & 6 & $6,9(4,0)$ & $6,0(4,0)$ \\
Limitações funcionais [0-36] & 9 & $7,1(6,1)$ & $4,9(3,7)$ \\
Bem-estar emocional [0-36] & 9 & $11,4(8,6)^{*}$ & $6,6(5,8)^{*}$ \\
Bem-estar social [0-52] & 13 & $7,7(8,2)^{*}$ & $4,4(4,9)^{*}$ \\
Percepção global & & & \\
Saúde bucal [0-4] & 1 & $2,6(0,9)^{*}$ & $2,1(0,8)^{*}$ \\
Bem-estar geral [0-4] & 1 & $1,1(1,1)$ & $0,8(0,9)$ \\
\hline
\end{tabular}

$\mathrm{CPQ}_{11-14}$ : Child Perceptions Questionnaire; [ ] possível variação do escore; *p<0,01 (teste Mann-Whitney) 
Tabela III - Matriz de correlação entre fatores socioeconômicos e o número de dentes cariados, perdidos/esfoliados e obturados (n=142). Carapicuíba - SP, 2012.

\begin{tabular}{lccccc}
\hline $\begin{array}{r}\mathbf{r} \\
\text { (p-valor) }\end{array}$ & $\begin{array}{c}\text { Número de } \\
\text { pessoas }\end{array}$ & $\begin{array}{c}\text { Número de } \\
\text { cômodos }\end{array}$ & $\begin{array}{c}\text { Escolaridade do } \\
\text { pai }\end{array}$ & $\begin{array}{c}\text { Escolaridade } \\
\text { da mãe }\end{array}$ & CPOD/ceo-d \\
\hline Renda & $-0,205$ & 0,268 & 0,145 & 0,216 & $-0,093$ \\
& $(0,034)^{*}$ & $(0,005)^{*}$ & $(0,144)$ & $(0,027)^{*}$ & $(0,327)$ \\
Número de pessoas & & 0,082 & $-0,025$ & $-0,232$ & 0,267 \\
& & $(0,368)$ & $(0,795)$ & $(0,013)^{*}$ & $(0,003)^{*}$ \\
Número de cômodos & & 0,0674 & $-0,046$ & $-0,042$ \\
& & $(0,477)$ & $(0,625)$ & $(0,640)$ \\
Escolaridade do pai & & & 0,323 & $-0,050$ \\
& & & & $(0,000)^{*}$ & $(0,587)$ \\
Escolaridade da mãe & & & & $-0,002$ \\
\end{tabular}

CPOD/ceo-d: número de dentes cariados, perdidos/esfoliados e obturados; * p $<0,05$ (teste de correlação de Spearman)

\section{DISCUSSÃO}

O presente estudo foi realizado com o objetivo de avaliar a relação da experiência de cárie na QVRSB em escolares da rede pública do município de Carapicuíba-SP, verificando-se também a relação entre o número de dentes cariados, perdidos/esfoliados e obturados e os fatores socioeconômicos.

Encontrou-se experiência de cárie em 58,5\% dos escolares avaliados no presente estudo e índice CPOD/ ceo-d de 2,1 - índice este superior à média encontrada na capital do estado de São Paulo no último levantamento epidemiológico em saúde bucal, que foi $1,41^{(3)}$. Na região Sudeste, esse levantamento encontrou experiência de cárie dentária em 51,5\% das crianças com 12 anos $^{(3)}$, índice este que diminuiu ao longo dos anos, mas demonstra que a atenção básica em saúde é ainda muito importante para diminuir os agravos à saúde bucal.

$\mathrm{Na}$ amostra avaliada da atual pesquisa, observou-se que o escore total obtido pelo instrumento $\mathrm{CPQ}_{11-14} \mathrm{em}$ escolares com experiência de cárie foi significativamente maior do que em crianças sem a experiência, bem como houve diferença de percepção global em saúde bucal entre os grupos. A relação entre cárie dentária e impacto na qualidade de vida está relacionada à experiência de dor, dificuldades de mastigação e fonação, e desconfortos estéticos, o que direta ou indiretamente afeta o dia a dia da criança e seus familiares $^{(5,13,20-22)}$. Entre crianças de 8 a 10 anos de escolas públicas e privadas de Pelotas-RS, a presença de dentes cariados também influenciou negativamente a QVRSB $^{(1)}$, demonstrando a importância de medidas preventivas e terapêuticas para a doença. $\mathrm{O} \mathrm{CPQ}_{11-14}$ avalia a percepção da própria criança sobre como sua saúde bucal influencia suas funções físicas e psicossociais ${ }^{(12)}$, fornecendo medidas que sirvam para o desenvolvimento de estratégias em saúde bucal $^{(5,14)}$.

Sabe-se que as manifestações vivenciadas por um indivíduo são determinadas não apenas pela natureza da doença, mas também pelas suas características pessoais e ambientais $^{(1,12-14,23)}$. Um estudo ${ }^{(24)}$ mostrou que crianças de 8 anos de idade foram capazes de reportar maiores impactos no seu bem-estar emocional devido às suas condições bucais, enquanto as crianças de 10 anos reportaram maiores impactos em seu bem-estar social por conta das suas condições bucais. A percepção difere entre idades diferentes e pode ser ainda mais diversa entre culturas e condições clínicas diferentes ${ }^{(1,25)}$. A avaliação individual da percepção de QVRSB dá a oportunidade de mensurar o quanto uma condição clínica é importante àquele indivíduo e qual o impacto que ele perceberia ao receber determinado tratamento, articulando a saúde com condições de vida e qualidade de vida, e priorizando as medidas de saúde para aqueles com qualidade de vida mais comprometida.

Crianças com saúde bucal prejudicada estão mais propensas a se sentirem preocupadas e chateadas com sua condição bucal, o que pode causar um impacto no domínio emocional $^{(12)}$ devido às limitações nas atividades diárias que acarretam privações em seu contexto individual, ambiental e social ${ }^{(1,12)}$. Esse aspecto foi observado no presente estudo, em que crianças com experiência de cárie apresentaram maiores escores no domínio "bem-estar emocional" em relação àquelas sem a experiência, de tal modo que a criança pode tornar-se retraída e triste pela própria situação em que se encontra ${ }^{(11)}$. 
Estudos prévios observaram impacto no domínio emocional causado pela doença cárie tanto ao utilizar o autorrelato $^{(14,26)}$ quanto o relato dos pais ${ }^{(22)}$. O autorrelato, instrumento utilizado no presente estudo, parece ser mais fidedigno na avaliação da qualidade de vida da criança do que o relato dos pais, já que este pode trazer como viés a falta de representatividade da realidade da criança e a pressão relacionada à aceitabilidade social ${ }^{(27)}$.

A maioria das doenças bucais não é fatal, mas causa morbidades que podem comprometer a qualidade de vida do individuo e impactar seu bem-estar social ${ }^{(7,12-14,20,22)}$. $\mathrm{Na}$ infância, a cárie dentária é considerada a doença mais comum dentre aquelas que não regridem espontaneamente nem são passíveis de cura, e pode ter como consequência insatisfação com sua aparência ${ }^{(5,7,12,14,20,22,26)}$. Uma aparência bucal insatisfatória pode ser motivo de constrangimento (bullying) $)^{(1)}$, já que a aparência é fundamental para a experiência social, e a reação das pessoas às diferenças faciais e dentárias visíveis pode gerar um julgamento social negativo para o indivíduo, afetando sua autoestima ${ }^{(28)}$. O presente estudo mostrou que crianças de 12 anos reportaram impactos substanciais no domínio "bem-estar social", corroborando com estudo prévio o qual também enfatizou que a presença de cárie pode comprometer as interpretações e considerações que a criança está formando a seu próprio respeito, prejudicando suas relações interpessoais ${ }^{(11)}$.

O estudo dos fatores de risco da cárie é relevante para o desenvolvimento de estratégias que possam diminuir sua incidência. Outros estudos encontraram forte associação entre fatores socioeconômicos e experiência de cárie, tais como renda, escolaridade dos pais, trabalho materno, número de moradores no domicílio, entre outros ${ }^{(14,29,30)}$. Um estudo conduzido em Recife-PE observou que morar em domicílio com seis ou mais pessoas e ter mais de três moradores por quarto tornava as crianças mais propensas à experiência de cárie ${ }^{(31)}$. O presente estudo também observou que o número de dentes cariados, perdidos e obturados correlacionou-se com o número de pessoas que habitavam o domicílio das crianças, o que provavelmente impacta de forma significativa o acesso à saúde, já que um número maior de moradores no domicílio pode estar relacionado a uma maior divisão da renda e dos cuidados com a saúde, resultando em privação material nas decisões diárias familiares ${ }^{(12)}$ e se refletindo nos hábitos de saúde praticados pela criança e sua família ${ }^{(14)}$.

Já a renda mensal familiar declarada pelos investigados da atual pesquisa não mostrou uma correlação significativa com o índice CPOD/ceo-d, provavelmente devido ao seu caráter variável e flutuante. Como a renda familiar foi autodeclarada, há uma tendência a enfatizar ou exagerar uma medida desejável pelo entrevistado, gerando uma possibilidade de viés nesse tipo de mensuração ${ }^{(32)}$.
Um fato importante a ser pontuado é que a amostra do presente estudo compreendeu crianças não referenciadas para tratamento odontológico que tenderiam a apresentar menor impacto na qualidade de vida relacionada à saúde do que crianças referenciadas. Sabe-se que a dor é uma das principais motivadoras para a procura por tratamento ${ }^{(33)}$; sendo assim, esses indivíduos reportariam o desconforto ou a limitação de forma mais precisa e intensa. Em outras condições clínicas, como a obesidade, os indivíduos que procuram tratamento, em geral, apresentam os piores sinais e sintomas ${ }^{(34)}$. Mesmo assim, os escores obtidos na escala total no grupo com experiência de cárie foi significativamente maior do que no grupo sem experiência no estudo em questão.

O desenho do presente estudo (corte transversal) limita, de certo modo, a generalização dos resultados obtidos. Nesse sentido, estudos prospectivos são necessários para entender em que extensão a saúde bucal prejudicada impacta a QVRSB de indivíduos em crescimento, se repercute para a fase adulta e se o tratamento dentário é capaz de melhorar sua qualidade de vida.

\section{CONCLUSÃO}

Os achados do presente estudo mostraram que nos escolares de 12 anos avaliados do município de Carapicuíba-SP, a experiência de cárie relacionou-se com a percepção negativa da saúde bucal, principalmente nos aspectos emocional e social, e com o número de pessoas que habitavam o domicílio da criança.

\section{AGRADECIMENTOS}

Autores agradecem ao apoio financeiro da Fundação de Amparo à Pesquisa do Estado de São Paulo (processo n. 2012/04492-2).

\section{REFERÊNCIAS}

1. Schuch HS, Santos Costa F, Torriani DD, Demarco FF, Goettems ML. Oral health-related quality of life of schoolchildren: impact of clinical and psychosocial variables. Int J Paediatr Dent. 2015;25(5):358-65.

2. Cooper AM, O'Malley LA, Elison SN, Armstrong R, Burnside G, Adair P, et al. Primary school-based behavioural interventions for preventing caries. Cochrane Database Syst Rev. 2013;5:CD009378.

3. Ministério da Saúde (BR). Projeto Saúde Bucal Brasil: levantamento nacional em saúde bucal 2010 [acesso em 2014 Maio 21]. Disponível em: http://189.28.128.100/ dab/docs/geral/apresentacao_SB2010.pdf. 2011 
4. Foster Page LA, Thomson WM, Ukra A, Baker SR. Clinical status in adolescents: is its impact on oral health-related quality of life influenced by psychological characteristics? Eur J Oral Sci. 2013;121(3 Pt 1):182-7.

5. Scarpelli AC, Paiva SM, Viegas CM, Carvalho AC, Ferreira FM, Pordeus IA. Oral health-related quality of life among Brazilian preschool children. Community Dent Oral Epidemiol. 2013;41(4):336-44.

6. Barbosa TS, Mialhe FL, Castilho ARF, Gavião MBD. Qualidade de vida e saúde bucal em crianças e adolescentes: aspectos conceituais e metodológicos. Physis. 2010;20(1):283-300.

7. Cohen-Carneiro F, Souza-Santos R, Rebelo MA. Quality of life related to oral health: contribution from social factors. Ciênc Saúde Coletiva. 2011;16(Suppl 1):1007-15.

8. Kumar S, Kroon J, Lalloo R. A systematic review of the impact of parental socio-economic status and home environment characteristics on children's oral health related quality of life. Health Qual Life Outcomes. 2014;12:41.

9. Barbosa TS, Gavião MBD. Qualidade de vida e saúde bucal em crianças - parte II: versão brasileira do Child Perceptions Questionnaire. Ciênc Saúde Coletiva. 2011;16(7):67-3276.

10. Barbosa TS, Castelo PM, Leme MS, Gavião MB. Associations between oral health-related quality of life and emotional statuses in children and preadolescents. Oral Dis. 2012;18(7):639-47.

11. Araújo AR, Santos MTBR, Duarte DA. O impacto da doença cárie na qualidade de vida em crianças de 08 a 10 anos. Arq Med Hosp Fac Cienc Med Santa Casa São Paulo. 2009;54(1):1-5.

12 .Piovesan C, Antunes JL, Guedes RS, Ardenghi TM. Impact of socioeconomic and clinical factors on child oral health-related quality of life (COHRQoL). Qual Life Res. 2010;19(9):1359-66.

13. Piovesan C, Antunes JL, Mendes FM, Guedes RS, Ardenghi TM. Influence of children's oral healthrelated quality of life on school performance and school absenteeism. J Public Health Dent. 2012;72(2):156-63.

14. Paula JS, Leite IC, Almeida AB, Ambrosano GM, Pereira AC, Mialhe FL. The influence of oral health conditions, socioeconomic status and home environment factors on schoolchildren's self-perception of quality of life. Health Qual Life Outcomes. 2012;10:6.

15. Lopes LM, Vazquez FL, Pereira AC, Romão DA. Indicadores e fatores de risco da cárie dentária em crianças no Brasil: uma revisão de literatura. RFO UPF. 2014;19(2):245-51.

16. Garcia RN, Almeida EB, Souza K, Vechi G. Nutrição e Odontologia: a prática interdisciplinar em um projeto de extensão. RSBO. 2008;5(1):50-7.

17. Instituto Brasileiro de Geografia e Estatística - IBGE. Perfil dos Municípios Brasileiros 2011 [acesso em 2014 Nov 10]. Disponível em: http://cidades.ibge.gov. br

18. Fundação Sistema Estadual de Análise de Dados do Estado de São Paulo - SEADE. Perfil Municipal Carapicuíba [acesso em 2014 Nov 10]. Disponível em: http://produtos.seade.gov.br/produtos/perfil/ perfilMunEstado.php

19. World Health Organization - WHO. Oral health surveys: basic methods. $4^{\text {th }}$ ed. Geneva: World Health Organization, 1997.

20. Gomes MC, Pinto-Sarmento TC, Costa EM, Martins CC, Granville-Garcia AF, Paiva SM. Impact of oral health conditions on the quality of life of preschool children and their families: a cross-sectional study. Health Qual Life Outcomes. 2014;12:55.

21. Navarro MF, Modena KC, Bresciani E. Social disparity and oral health. Braz Oral Res. 2012;26 Suppl 1:17-24.

22. Onoriobe U, Rozier RG, Cantrell J, King RS. Effects of enamel fluorosis and dental caries on quality of life. J Dent Res. 2014;93(10):972-9.

23. Rodd HD, Marshman Z, Porritt J, Bradbury J, Baker SR. Oral health-related quality of life of children in relation to dental appearance and educational transition. Br Dent J. 2011;22;211(2):E4.

24. Jokovic A, Locker D, Tompson B, Guyatt G. Questionnaire for measuring oral health-related quality of life in eight- to ten-year-old children. Pediatr Dent. 2004;26(6):512-8.

25. Gordia AP, Quadros TM, Campos WD. Sociodemographic variables as determinant of the environment domain of quality of life of adolescents. Ciênc Saúde Coletiva. 2009;14(6):2261-8.

26. Krisdapong S, Prasertsom P, Rattanarangsima K, Sheiham A. Impacts on quality of life related to dental caries in a national representative sample of Thai 12and 15-year-olds. Caries Res. 2013;47(1):9-17.

27. McGrath C, Broder H, Wilson-Genderson M. Assessing the impact of oral health on the life quality of children: implications for research and practice. Community Dent Oral Epidemiol. 2004;32(2):81-5. 
28. Wilson IB, Cleary PD. Linking clinical variables with health-related quality of life. A conceptual model of patient outcomes. JAMA. 1995;273(1):59-65.

29. Lopes RM, Domingues GG, Junqueira SR, Araujo ME, Frias AC. Conditional factors for untreated caries in 12-year-old children in the city of São Paulo. Braz Oral Res. 2013;27(4):376-81.

30. Feldens CA, Giugliani ER, Vigo Á, Vítolo MR. Early feeding practices and severe early childhood caries in four-year-old children from southern Brazil: a birth cohort study. Caries Res. 2010;44(5):445-52.

31. Melo MM, Souza WV, Lima ML, Braga C. Factors associated with dental caries in preschoolers in Recife, Pernambuco State, Brazil. Cad Saúde Pública. 2011;27(3):471-85.

32. Loughnan S, Kuppens P, Allik J, Balazs K, de Lemus $\mathrm{S}$, Dumont K, et al. Economic inequality is linked to biased self-perception. Psychol Sci. 2011;22(10):12548 .

33. Morlin MT, Lemos JBD. Escalas subjetivas de dor: breve análise da literatura e considerações sobre seu uso em clínica e pesquisa odontológica. J Bras Ocl ATM Dor Orofac. 2005;19(5):66-74.
34. Van Vlierberghe L, Braet C, Goossens L, Mels S. Psychiatric disorders and symptom severity in referred versus non-referred overweight children and adolescents. Eur Child Adolesc Psychiatry. 2009;18(3):164-73.

\section{Endereço do primeiro autor:}

Ednalva Maria de Sousa Eskenazi

Av. Presidente Vargas, 280

Bairro: Vila Caldas

CEP: 06310-100 - Carapicuíba - SP - Brasil

E-mail: ednaeskenazi@uol.com.br

\section{Endereço para correspondência:}

Profa. Paula Midori Castelo

Universidade Federal de São Paulo - UNIFESP

Departamento de Ciências Biológicas, Campus Diadema

Rua São Nicolau, 210/1 Andar

Bairro: Centro

CEP: 09913-030 - Diadema - SP - Brasil

E-mail: pcastelo@yahoo.com 